\title{
Personality and suicide risk: the impact of economic crisis in Japan
}

\author{
F. Tanji ${ }^{1,2}$, M. Kakizaki ${ }^{1}$, Y. Sugawara ${ }^{1 *}$, I. Watanabe ${ }^{1,3}$, N. Nakaya ${ }^{4}$, Y. Minami $^{2}$, A. Fukao ${ }^{5}$ and \\ I. Tsuji ${ }^{1}$ \\ ${ }^{1}$ Division of Epidemiology, Department of Public Health and Forensic Medicine, Tohoku University Graduate School of Medicine, Sendai, Miyagi, \\ Japan \\ ${ }^{2}$ Division of Community Health, Tohoku University Graduate School of Medicine, Sendai, Miyagi, Japan \\ ${ }^{3}$ Department of Health Sciences, Tohoku University Graduate School of Medicine, Sendai, Miyagi, Japan \\ ${ }^{4}$ Department of Preventive Medicine and Epidemiology, Tohoku Medical Megabank Organization, Tohoku University, Sendai, Miyagi, Japan \\ ${ }^{5}$ Department of Public Health, Yamagata University Graduate School of Medical Science, Yamagata, Japan
}

Background. The interactive effect of personal factors and social factors upon suicide risk is unclear. We conducted prospective cohort study to investigate whether the impact of the economic crisis in 1997-1998 upon suicide risk differed according to Neuroticism and Psychoticism personality traits.

Methods. The Miyagi Cohort Study in Japan with a follow-up for 19 years from 1990 to 2008 has 29432 subjects aged 40-64 years at baseline who completed a questionnaire about various health habits and the Japanese version of the Eysenck Personality Questionnaire - Revised Short Form in 1990.

Results. The suicide mortality rate increased from 4.6 per 100000 person-years before 1998 to 27.8 after 1998 . Although both Neuroticism and Psychoticism were significantly associated with an increased risk of mortality during the whole period from 1990 to 2008, the impact of the economic crisis upon suicide risk differed between the Neuroticism and Psychoticism personality traits. Compared with the lowest category, the hazard ratios (HRs) for the highest Neuroticism increased from 0.66 before 1998 to 2.45 after 1998. On the other hand, the HRs for the highest Psychoticism decreased from 7.85 before 1998 to 2.05 after 1998.

Conclusions. The impact of the 1997-1998 economic crisis upon suicide risk differed according to personality. Suicide risk increased among these with higher Neuroticism after the economic crisis, but this was not the case for other personality subscales.

Received 27 February 2013; Revised 17 June 2014; Accepted 20 June 2014; First published online 18 July 2014

Key words: Cohort study, economic crisis, Japan, personality, suicide risk.

\section{Introduction}

Economic difficulty is one of the most important causes of suicide (Lewis \& Sloggett, 1998; Inoue et al. 2007; Stuckler et al. 2009). The economic crisis in 1997-1998 harmed the economies of many Asian countries, and resulted in a sharp increase of suicide mortality (Chang et al. 2009; Kim et al. 2011). Until 1997 in Japan, the suicide rate had ranged from 16.1 to 18.8 per 100000 people. However, in 1998, it jumped to 25.4, and since then has remained around 25.0 (Statistics and Information Department Minister's

* Address for correspondence: Y. Sugawara, Ph.D., Division of Epidemiology, Department of Public Health and Forensic Medicine, Tohoku University Graduate School of Medicine, 2-1 Seryo-machi, Aoba-ku, Sendai, Miyagi, 980-8575, Japan.

(Email: yumi1717@med.tohoku.ac.jp)
Secretariant Ministry of Health Labour and Welfare Japan, 2011).

The risk factors for suicide are not only economic, but also psychological, physical, and cultural (Bertolote \& Fleischmann, 2005). Yoshimasu et al. (2008) divided these risk factors into two categories, personal and social factors, and they assumed that the interactive effects of these two factors may affect the suicidal risks.

Previous studies have agreed that personality traits such as Neuroticism and Psychoticism in the Eysenck Personality Questionnaire (EPQ) are associated with an increased risk of suicide (Lolas et al. 1991; Duberstein et al. 1994; Velting, 1999; Useda et al. 2004; Tsoh et al. 2005; Brezo et al. 2006). Since personality determines a person's response to life events (Kendler et al. 2004), we may hypothesize that the impact of economic difficulty upon suicide risk must differ according to personality traits. However, no study 
has investigated the interactive effects of economic difficulty and personality upon suicide risk.

The objective of this study was to investigate whether the relationship between personality and suicide differed before and after the economic crisis in 1997-1998. This knowledge would lead us to further understanding of the mechanisms how different personality traits cope with social stress, thus contributing to the planning of effective suicide prevention. For this purpose, we analysed data from a population-based prospective cohort study in Japan, which we have been following up since 1990. Our cohort is unique in that it can analyse the association between personality and suicide risk, both before the economic crisis (1990-1997) and after the crisis (1998-2008), separately.

\section{Methods}

\section{Study cohort}

The design of the Miyagi Cohort Study has been described in detail elsewhere (Fukaol et al. 1995; Nakaya et al. 2005). In brief, we delivered two selfadministered questionnaires to all 51921 residents (25279 men and 26642 women) aged 40-64 years in 14 municipalities of Miyagi Prefecture, northern Japan, between June and August 1990. The first questionnaire asked about various health habits, and the second was the Japanese version of the Eysenck Personality Questionnaire - Revised (EPQ-R) Short Form (Hosokawa \& Ohyama, 1993). The questionnaires were delivered to, and collected from, the subjects' residences by members of Health Promotion Committees appointed by the municipal government. We excluded one subject because of withdrawal before June $1990(n=51920)$. The response rate for the first questionnaire was $91.7 \%(n=47604)$, and that for the second questionnaire among the respondents to the first was $87.0 \%(n=41423)$. The study protocol was approved by the institutional review board of Tohoku University School of Medicine. We considered the return of self-administered questionnaires signed by the participants to imply their consent to participate in the study.

\section{Exposure data}

The EPQ-R has 48 questions with dichotomized responses (yes or no), and scores for each of the four subscales (Extraversion, Neuroticism, Psychoticism, Lie) are calculated on the basis of 12 questions each. The scores on each subscale range from 0 to 12 , with higher scores indicating a greater tendency to possess the personality trait represented by each subscale. Extraversion represents sociability, liveliness and surgency; Neuroticism represents emotional instability and anxiousness; Psychoticism represents toughmindedness, aggressiveness, coldness, and egocentricity; Lie represents unsophisticated dissimulation and social naivety or conformity (Eysenck \& Eysenck, 1991).

Several of Eysenck's personality questionnaires have been translated into Japanease (Hosokawa \& Ohyama, 1993). In previous work, Hosokawa et al. developed the Japanese version of the EPQ-R and examined its reproducibility and validity among 329 college students and 253 adults (Hosokawa \& Ohyama, 1993). Cronbach's $\alpha$ coefficient, a measure of internal consistency, was $>0.70$ for all subscales except Psychoticism ( 0.42 for college students and 0.48 for adults). Testretest reliability coefficients of the four subscales over a 6-month period ranged from 0.70 to 0.85 , indicating substantial stability. Confirmatory factor analysis supported the original theoretical structure of the four scales proposed by Eysenck and colleagues.

\section{Follow-up}

The endpoint was suicide mortality. To follow up the participants for mortality and migration, we established a Follow-up Committee (Tsuji et al. 2004). This Committee consisted of the Miyagi Cancer Society, the Community Health Divisions of all 14 municipalities, the Department of Health and Welfare of Miyagi Prefectural Government, and the Division of Epidemiology, Tohoku University Graduate School of Medicine. The Committee periodically reviewed the Residential Registration Record of each municipality. With this review, we identified participants who had either died or emigrated during the followup period. We discontinued follow-up of those who had emigrated from the study area, because the Committee could not review the Residential Registration Record outside the study area.

For decedents, we investigated the causes of death by reviewing the death certificates with permission from the Ministry of Health, Labour and Welfare, Japan. Cause of death was classified according to the International Classification of Diseases (ICD), 9th revision, between 1 June 1990 and 31 December 1998 (WHO, 1977), and 10th revision, between 1 January 1999 and 31 December 2008 (WHO, 1992). Death due to suicide was identified as ICD-9: E950-E959, or ICD-10: X60-X84.

Of the 41423 subjects who responded to the two questionnaires, we excluded 54 subjects who responded 'yes' or 'no' to all 48 items and 8600 subjects for whom responses to any of the 48 items in the EPQ-R were missing. We also excluded 2493 subjects who indicated that the two questionnaires had been completed by other family members, because we 


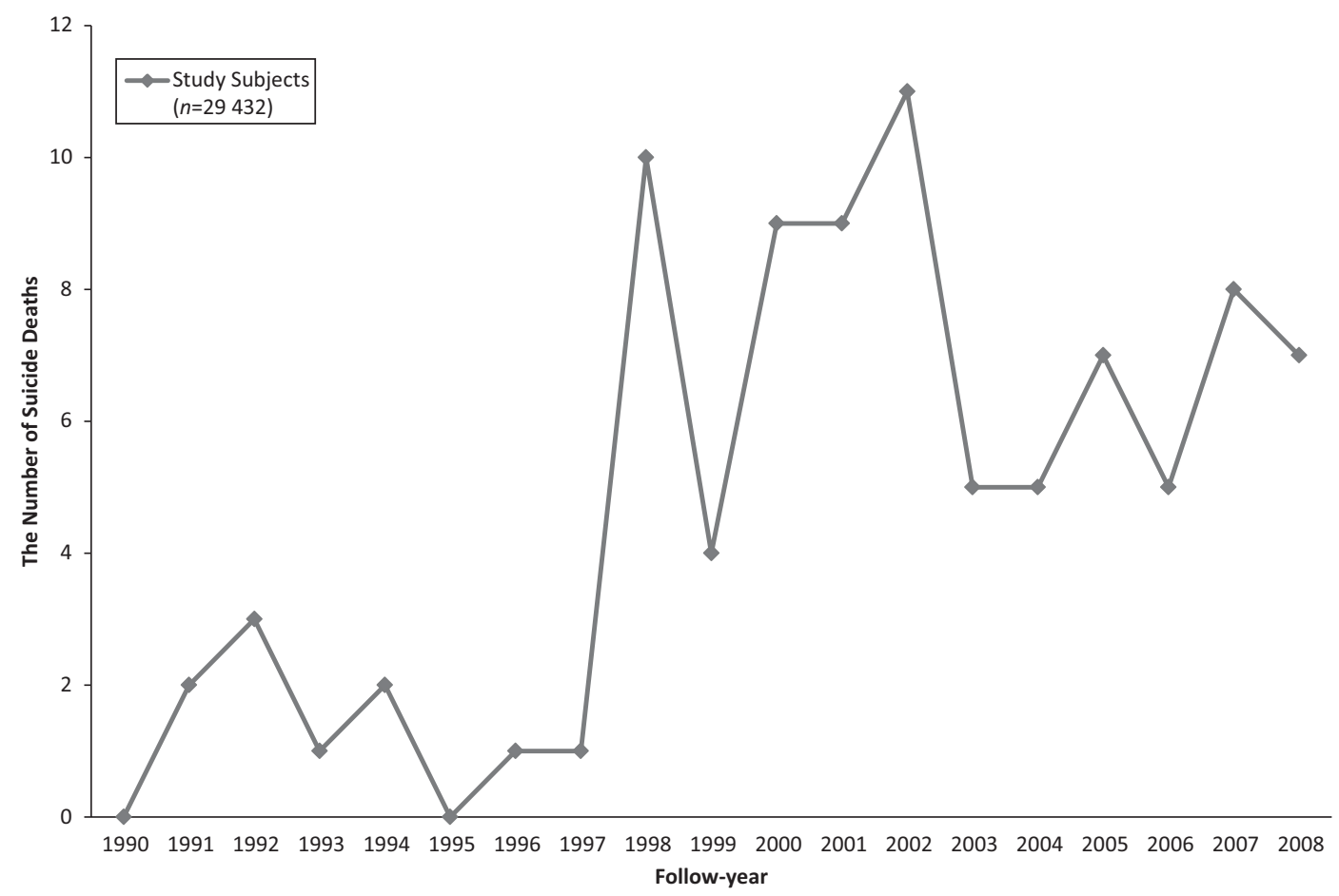

Fig. 1. Annual trend for the number of suicide deaths 1990-2008 (The Miyagi Cohort Study).

considered that such aid might have affected the subjects' response patterns. In addition, we excluded 844 subjects who had entered a history of cancer, stroke, or myocardial infarction in the self-reported questionnaire. Finally, 29432 subjects (14327 men and 15105 women) were used for the final analysis.

We counted person-years of follow-up for each subject from 1 June 1990 until the date of death, the date of emigration from the study districts, or the end of follow-up, whichever occurred first. A total of 504595 person-years resulted. Among the 29432 subjects, the number of deaths due to suicide was 90 (suicide rate 17.8 per 100000 people). A total of 1892 subjects $(6.4 \%$ of the analytic cohort) were lost to follow-up during the study period.

\section{Statistical analysis}

Each personality subscale was divided into four categories to distribute the total subjects as closely as possible into even-sized quartiles. We calculated the annual trend for the number of suicide deaths between 1990 and 2008 in the study subjects $(n=29432)$. We used the Cox proportional hazards model to calculate the hazard ratios (HRs) and 95\% confidence intervals (CIs) for suicide mortality according to each category of a personality subscale, the lowest category being treated as the reference group, and to adjust for potentially confounding variables. In the case of missing values for a confounding variable, we created a separate missing category and included this in the models. In addition, we conducted these analyses separately by sex (data not shown). Trend tests were performed by treating personality subscales as continuous variables.

In these analyses, we regarded the following variables as potential confounders: age (continuous variable), sex, history of hypertension or diabetes mellitus (presence or absence), smoking status (never, former, currently smoking 1-19 cigarettes/day, or $\geqslant 20$ cigarettes/day), alcohol consumption (never, former, current ethanol intake of $<23.0$, or $\geqslant 23.0 \mathrm{~g} /$ day), body mass index in $\mathrm{kg} / \mathrm{m}^{2}(\leqslant 18.4,18.5-24.9$, or $\geqslant 25.0)$, green tea consumption ( $<1$ cup/day, 1-2 cups/day, or $\geqslant 3$ cups/day), education (in school until age $£ 15$ years, age $16-18$ years, or age $\geqslant 19$ years), marital status (whether or not living with spouse), sleep duration $(\leqslant 6,7-8$, or $\geqslant 9 \mathrm{~h} /$ day $)$, time spent walking $(\leqslant 0.5$, $0.5-1$, or $\geqslant 1 \mathrm{~h} /$ day), ikigai (having, uncertain, or not having), stress (high, moderate, or low).

In Japanese culture, having ikigai (sense of 'life worth living') is the most commonly used indicator of subjective well-being. A Japanese study has reported that subjects without a sense of ikigai were significantly associated with an increased risk of all-cause mortality (Sone et al. 2008).

In addition, we repeated the analyses after excluding all deaths that occurred within the first 3 years of follow-up, because subjects who died during this period might have been in poor health at the baseline. 
Table 1. Characteristics of the subjects according to the highest and lowest categories of four personality subscales, the Miyagi Cohort, Japan, 1990-2008

\begin{tabular}{|c|c|c|c|c|c|c|c|c|}
\hline \multirow[b]{3}{*}{ Characteristics } & \multirow{2}{*}{\multicolumn{2}{|c|}{ Extraversion }} & \multirow{2}{*}{\multicolumn{2}{|c|}{ Neuroticism }} & \multirow{2}{*}{\multicolumn{2}{|c|}{ Psychoticism }} & \multirow{2}{*}{\multicolumn{2}{|c|}{ Lie }} \\
\hline & & & & & & & & \\
\hline & $\leqslant 3$ & $\geqslant 9$ & $\leqslant 3$ & $\geqslant 8$ & $\leqslant 2$ & $\geqslant 5$ & $\leqslant 5$ & $\geqslant 10$ \\
\hline Number of subjects & 8694 & 6151 & 8495 & 8381 & 9551 & 6863 & 7177 & 5980 \\
\hline Mean age, years (S.D.) & $50.8(7.5)$ & $51.1(7.4)$ & $51.4(7.6)$ & $50.6(7.5)$ & $51.9(7.6)$ & $49.8(7.3)$ & $48.0(6.9)$ & $54.3(7$. \\
\hline Women $(\%)$ & 53.2 & 47.7 & 47.4 & 54.0 & 65.3 & 32.0 & 39.3 & 57.0 \\
\hline Past history of diseases (\%) & 21.5 & 20.1 & 18.4 & 22.7 & 22.0 & 18.7 & 18.1 & 23.6 \\
\hline \multicolumn{9}{|l|}{ Smoking status (\%) } \\
\hline Never smoked & 48.5 & 41.6 & 44.1 & 46.5 & 55.3 & 32.1 & 39.0 & 49.0 \\
\hline Smoked in the past & 10.3 & 10.1 & 9.8 & 10.6 & 8.6 & 12.4 & 11.9 & 8.9 \\
\hline Currently smoking & 29.5 & 37.4 & 35.2 & 30.3 & 21.7 & 47.5 & 43.0 & 25.6 \\
\hline Missing & 11.7 & 10.9 & 10.9 & 12.6 & 14.4 & 8.0 & 6.1 & 16.5 \\
\hline \multicolumn{9}{|l|}{ Drinking status (\%) } \\
\hline Never drunk alcohol & 43.3 & 30.0 & 36.0 & 37.4 & 45.8 & 27.2 & 29.2 & 43.2 \\
\hline Drank in the past & 5.1 & 4.4 & 4.3 & 5.5 & 4.2 & 5.8 & 5.1 & 4.8 \\
\hline Currently drinking & 42.8 & 57.5 & 51.2 & 48.1 & 39.5 & 60.7 & 61.0 & 39.7 \\
\hline Missing & 8.8 & 8.1 & 8.5 & 9.0 & 10.5 & 6.3 & 4.7 & 12.3 \\
\hline \multicolumn{9}{|l|}{ BMI $\left(\mathrm{kg} / \mathrm{m}^{2}\right)(\%)$} \\
\hline$\leqslant 18.4$ & 3.2 & 1.5 & 1.5 & 3.1 & 2.6 & 2.1 & 2.4 & 2.3 \\
\hline $18.5-24.9$ & 69.6 & 62.3 & 63.8 & 68.3 & 67.4 & 65.7 & 67.6 & 66.4 \\
\hline$\leqslant 25.0$ & 24.2 & 32.9 & 31.6 & 25.1 & 27.1 & 28.4 & 27.5 & 27.1 \\
\hline Missing & 3.1 & 3.3 & 3.1 & 3.5 & 2.9 & 3.8 & 2.5 & 4.2 \\
\hline \multicolumn{9}{|l|}{ Green tea consumption (\%) } \\
\hline$<1$ cup/day & 28.1 & 24.2 & 23.9 & 27.7 & 25.3 & 27.5 & 27.8 & 23.6 \\
\hline $1-2$ cups/day & 21.6 & 20.3 & 21.9 & 21.6 & 20.9 & 21.3 & 23.2 & 20.3 \\
\hline$\leqslant 3 \mathrm{cups} /$ day & 40.0 & 45.4 & 44.0 & 39.6 & 44.0 & 39.7 & 40.6 & 43.5 \\
\hline Missing & 10.3 & 10.1 & 10.2 & 11.1 & 9.8 & 11.5 & 8.4 & 12.6 \\
\hline \multicolumn{9}{|l|}{ Education level (\%) } \\
\hline$\leqslant 15$ years & 36.1 & 31.8 & 33.4 & 34.5 & 33.3 & 35.2 & 29.1 & 38.5 \\
\hline $16-18$ years & 44.6 & 47.2 & 46.2 & 45.7 & 47.6 & 44.6 & 49.5 & 42.8 \\
\hline$\geqslant 19$ years & 13.9 & 16.3 & 15.2 & 14.1 & 14.3 & 14.1 & 16.9 & 12.7 \\
\hline Missing & 5.4 & 4.7 & 5.2 & 5.7 & 4.8 & 6.1 & 4.5 & 6.0 \\
\hline
\end{tabular}




\begin{tabular}{|c|c|c|c|c|c|c|c|c|}
\hline \multicolumn{9}{|l|}{ Marital status (\%) } \\
\hline Living with spouse & 81.6 & 85.4 & 83.3 & 82.9 & 82.9 & 83.2 & 87.0 & 79.2 \\
\hline Not living with spouse & 9.8 & 7.6 & 8.2 & 9.3 & 9.2 & 8.6 & 7.7 & 10.0 \\
\hline Missing & 8.6 & 7.0 & 8.5 & 7.8 & 7.9 & 8.2 & 5.3 & 10.8 \\
\hline \multicolumn{9}{|l|}{ Sleep duration (\%) } \\
\hline$\leqslant 6 \mathrm{~h}$ & 20.3 & 20.8 & 16.8 & 24.2 & 21.5 & 20.0 & 22.0 & 17.4 \\
\hline $7-8 \mathrm{~h}$ & 71.0 & 71.0 & 74.0 & 67.7 & 71.0 & 70.1 & 70.8 & 71.6 \\
\hline$\geqslant 9 h$ & 6.4 & 6.3 & 7.0 & 5.8 & 5.6 & 7.3 & 5.7 & 8.3 \\
\hline Missing & 2.3 & 1.9 & 2.2 & 2.3 & 1.9 & 2.6 & 1.5 & 2.7 \\
\hline \multicolumn{9}{|l|}{ Walking (\%) } \\
\hline$<0.5$ h/day & 32.1 & 26.7 & 26.3 & 32.5 & 27.9 & 31.7 & 32.9 & 24.8 \\
\hline $0.5-1 \mathrm{~h} /$ day & 22.8 & 24.3 & 24.3 & 22.9 & 24.8 & 22.1 & 24.9 & 22.1 \\
\hline$>1$ h/day & 38.3 & 43.9 & 43.3 & 38.6 & 41.2 & 39.5 & 38.9 & 45.1 \\
\hline Missing & 6.8 & 5.1 & 6.1 & 6.0 & 6.1 & 6.7 & 3.3 & 8.0 \\
\hline \multicolumn{9}{|l|}{ Ikigai (\%) } \\
\hline Having & 43.6 & 75.9 & 67.8 & 48.2 & 59.4 & 56.2 & 54.6 & 66.6 \\
\hline Uncertain & 48.8 & 20.4 & 27.8 & 44.1 & 35.6 & 36.5 & 40.1 & 27.9 \\
\hline Not having & 4.6 & 1.4 & 1.4 & 4.9 & 2.1 & 4.2 & 3.3 & 1.9 \\
\hline Missing & 3.0 & 2.3 & 3.0 & 2.8 & 2.9 & 3.1 & 2.0 & 3.6 \\
\hline \multicolumn{9}{|l|}{ Perceived mental stress (\%) } \\
\hline High & 27.0 & 17.8 & 7.0 & 40.5 & 23.4 & 20.2 & 26.6 & 15.1 \\
\hline Medium & 62.3 & 61.0 & 66.5 & 53.1 & 63.8 & 61.4 & 61.3 & 65.1 \\
\hline Low & 9.1 & 19.7 & 24.9 & 4.6 & 11.3 & 16.3 & 10.8 & 17.6 \\
\hline Missing & 1.6 & 1.5 & 1.6 & 1.8 & 1.5 & 2.1 & 1.3 & 2.2 \\
\hline \multicolumn{9}{|l|}{ Type of job (\%) } \\
\hline Employed by company & 23.6 & 24.4 & 22.8 & 24.3 & 21.4 & 26.7 & 30.1 & 17.5 \\
\hline Employed by government $^{\mathrm{a}}$ & 9.9 & 8.8 & 9.3 & 9.5 & 9.5 & 9.2 & 11.7 & 6.8 \\
\hline Primary industry ${ }^{\mathrm{b}}$ & 12.6 & 17.5 & 15.5 & 13.5 & 12.0 & 18.1 & 17.2 & 12.9 \\
\hline $\begin{array}{l}\text { Self-employed, or freelance } \\
\text { professional }\end{array}$ & 19.5 & 19.0 & 21.6 & 18.7 & 19.1 & 20.8 & 15.6 & 25.1 \\
\hline $\begin{array}{l}\text { Housewife, unemployed, } \\
\text { or other }\end{array}$ & 24.8 & 22.0 & 22.2 & 24.5 & 28.3 & 16.7 & 18.7 & 27.2 \\
\hline Missing & 9.6 & 8.3 & 8.6 & 9.5 & 9.7 & 8.5 & 6.7 & 10.5 \\
\hline
\end{tabular}

S.D., Standard deviation; BMI, body mass index.

${ }^{a}$ Medical care personnel, teacher, and civil service.

${ }^{\mathrm{b}}$ Agriculture, fishing, and forestry. 
Furthermore, we calculated the HRs and 95\% CIs for suicide mortality by dividing the follow-up period into two separate terms (from 1990 to 1997, and from 1998 to 2008). In addition, we examined in detail potential confounding and effect modification by age and other covariates on the associations between personality scales and suicide mortality. No statistically significant interaction was observed between personality and other confounding factors for suicide mortality on a multiplicative scale (data not shown).

All statistical analyses were performed using the SAS software package, v. 9.2 (SAS Institute Inc., USA). All reported $p$ values for analysis of linear trends were two-sided, and were considered statistically significant if $<0.05$.

\section{Results}

Fig. 1 shows the annual trend for the number of suicide deaths among the study subjects $(n=29432)$. The suicide mortality rate increased after 1998 by 6-fold relative to before 1998: from 4.6 to 27.8 per 100000 person-years.

Baseline characteristics according to the highest and lowest categories (i.e. approximate quartiles) of each personality subscale are shown in Table 1. Higher scores for Extraversion were associated with older age, being male, being having smoked or having drunk alcohol, being overweight, being married, having ikigai and not having high stress. Higher scores for Neuroticism were associated with younger age, being female, having a history of hypertension or diabetes mellitus, drinking green tea less often, short sleep duration, not having ikigai, and having high stress. Higher scores for Psychoticism were associated with having smoked or having drunk alcohol, being regularly employed by a company more often, and being a housewife, unemployed or in other industry less often. Higher scores for Lie were associated with older age, being female, having a history of hypertension or diabetes mellitus, never having smoked or never having drunk alcohol, feeling ikigai more often, marrying less often, walking more often, and having high stress less often.

Table 2 shows the association between personality subscales and suicide mortality during the whole study period of 1990-2008. Among the four personality traits, only Neuroticism and Psychoticism were significantly associated with suicide risk. For Neuroticism, higher category was associated with a significantly increased risk of suicide, compared with the lowest category (HR 2.04, 95\% CI 1.12-3.72, $p=0.003$ for trend). For Psychoticism, the highest category was associated with a significantly increased risk of suicide, compared with the lowest category
(HR 2.30, 95\% CI 1.28-4.15, $p=0.02$ for trend). We further divided the subjects into four groups on the basis of the same range of Personality Subscale scores $(0-3,4-6,7-9,10-12)$, and obtained quite similar results (data not shown). We also modelled each Personality Subscale score as a continuous variable, and calculated the relative risk of suicide mortality for each 1-point increment of the subscale. This showed that the risk of suicide mortality was significantly associated with Neuroticism (HR 1.12, 95\% CI 1.04-1.21) and Psychoticism (HR 1.15, 95\% CI 1.02-1.29) but not with Extraversion or Lie. These findings remained unchanged even after excluding all deaths that occurred within the first 3 years of follow-up. Similar results were obtained among men and women, respectively.

Table 3 shows the HRs and 95\% CIs for suicide mortality for the two separate periods (from 1990 to 1997, and from 1998 to 2008). Fig. 2 graphically demonstrated the same data as for Table 3. The association between Neuroticism and suicide risk became evident only after 1998. Between 1990 and 1997, a higher Neuroticism score was associated with a nonsignificantly decreased risk.

After 1998, however, the highest category of Neuroticism became a significant factor associated with increased risk (HR 2.45, 95\% CI 1.26-4.74, $p=0.003$ for trend). For Psychoticism, the risk for suicide mortality was significantly increased at the highest category in both 1990-1997 (HR 7.85) and 1998-2008 (HR 2.05). The impact of Psychoticism on suicide risk became attenuated after 1998. For Extraversion and Lie, the association with suicide mortality after 1998 remained unchanged in comparison with that before 1998 .

The above findings might be explained by the association between personality and occupation, because a previous study reported that cause-specific mortality in Japan was different among the types of job (Wada et al. 2012). In addition, Dupre et al. (2012) also reported that unemployment status is a risk of acute myocardial infarction. Thus, we considered the association between personality and types of job.

Table 1 shows the distribution of job types, and as can be seen, the distribution differed slightly according to personality. Subjects with higher Psychoticism scores were more likely to be employed by a company, and thus their employment status was less stable than in government or primary industry. However, there was no significant association between suicide mortality risk and job type. The HRs and 95\% CIs were 1.05 (0.55-2.01), 1.12 (0.56-2.25), 1.26 (0.62-2.54), and $1.05(0.48-2.31)$ for subjects who were employed by a company, primary industry, self-employed or freelance professional, and other (housewife, unemployed 
Table 2. Hazard ratios and 95\% confidence intervals for risk of death from suicide, the Miyagi Cohort, Japan, 1990-2008

\begin{tabular}{|c|c|c|c|c|c|c|}
\hline \multirow[b]{2}{*}{ Extraversion } & \multicolumn{4}{|c|}{ Personality subscale score levels } & \multirow[t]{2}{*}{ Per 1 point of score ${ }^{a}$} & \multirow[t]{2}{*}{$p$ trend $^{\mathrm{b}}$} \\
\hline & $\leqslant 3$ & $4-5$ & $6-8$ & $\geqslant 9$ & & \\
\hline No. of subjects & 8694 & 6446 & 8141 & 6151 & & \\
\hline No. of deaths & 23 & 25 & 22 & 20 & & \\
\hline Person-years of follow-up & 149229 & 110868 & 139071 & 105427 & & \\
\hline Age and sex adjusted HR $(95 \% \mathrm{CI})$ & 1.00 (ref.) & $1.47(0.84-2.60)$ & $1.01(0.57-1.82)$ & $1.16(0.64-2.11)$ & $1.01(0.94-1.08)$ & 0.92 \\
\hline Multivariable HR1 ${ }^{\mathrm{c}}(95 \% \mathrm{CI})$ & 1.00 (ref.) & $1.59(0.90-2.81)$ & $1.14(0.63-2.06)$ & $1.37(0.74-2.56)$ & $1.03(0.96-1.10)$ & 0.51 \\
\hline Multivariable HR2 ${ }^{\mathrm{d}}(95 \% \mathrm{CI})$ & 1.00 (ref.) & $1.40(0.77-2.56)$ & $1.19(0.65-2.17)$ & $1.36(0.72-2.58)$ & $1.03(0.96-1.11)$ & 0.44 \\
\hline Neuroticism & $\leqslant 3$ & $4-5$ & $6-7$ & $\geqslant 8$ & & \\
\hline No. of subjects & 8495 & 6365 & 6191 & 8381 & & \\
\hline No. of deaths & 18 & 18 & 15 & 39 & & \\
\hline Person-years of follow-up & 146275 & 109540 & 105698 & 143081 & & \\
\hline Age and sex adjusted HR $(95 \% \mathrm{CI})$ & 1.00 (ref.) & $1.41(0.73-2.71)$ & $1.23(0.62-2.44)$ & $2.39(1.37-4.18)$ & $1.14(1.06-1.22)$ & 0.0003 \\
\hline Multivariable HR1 ${ }^{\mathrm{c}}(95 \% \mathrm{CI})$ & 1.00 (ref.) & $1.31(0.68-2.54)$ & $1.10(0.55-2.20)$ & $2.04(1.12-3.72)$ & $1.12(1.04-1.21)$ & 0.003 \\
\hline Multivariable HR2 ${ }^{\mathrm{d}}(95 \% \mathrm{CI})$ & 1.00 (ref.) & $1.58(0.79-3.15)$ & $1.21(0.58-2.55)$ & $2.26(1.19-4.30)$ & $1.12(1.04-1.21)$ & 0.02 \\
\hline Psychoticism & $\leqslant 2$ & 3 & 4 & $\geqslant 5$ & & \\
\hline No. of subjects & 9551 & 7169 & 5849 & 6863 & & \\
\hline No. of deaths & 17 & 14 & 18 & 41 & & \\
\hline Person-years of follow-up & 164771 & 123038 & 100390 & 116396 & & \\
\hline Age and sex adjusted HR $(95 \% \mathrm{CI})$ & 1.00 (ref.) & $0.96(0.47-1.95)$ & $1.38(0.70-2.68)$ & $2.30(1.28-4.13)$ & $1.15(1.02-1.30)$ & 0.001 \\
\hline Multivariable HR1 ${ }^{\mathrm{c}}(95 \% \mathrm{CI})$ & 1.00 (ref.) & $0.97(0.47-1.97)$ & $1.36(0.70-2.67)$ & $2.30(1.28-4.15)$ & $1.15(1.02-1.29)$ & 0.002 \\
\hline Multivariable HR2 $^{\mathrm{d}}(95 \% \mathrm{CI})$ & 1.00 (ref.) & $0.81(0.39-1.71)$ & $1.18(0.59-2.36)$ & $2.06(1.14-3.73)$ & $1.12(0.99-1.27)$ & 005 \\
\hline Lie & $\leqslant 5$ & $6-7$ & $8-9$ & $\geqslant 10$ & & \\
\hline No. of subjects & 7177 & 7378 & 8897 & 5980 & & \\
\hline No. of deaths & 33 & 22 & 21 & 14 & & \\
\hline Person-years of follow-up & 122423 & 127046 & 152811 & 102315 & & \\
\hline Age and sex adjusted HR $(95 \% \mathrm{CI})$ & 1.00 (ref.) & $0.76(0.44-1.32)$ & $0.66(0.38-1.16)$ & $0.68(0.35-1.30)$ & $0.93(0.86-1.01)$ & 0.15 \\
\hline Multivariable HR1 ${ }^{\mathrm{c}}(95 \% \mathrm{CI})$ & 1.00 (ref.) & $0.78(0.45-1.35)$ & $0.70(0.39-1.23)$ & $0.76(0.39-1.48)$ & $0.94(0.87-1.02)$ & 0.28 \\
\hline Multivariable HR2 ${ }^{\mathrm{d}}(95 \% \mathrm{CI})$ & 1.00 (ref.) & $0.75(0.43-1.32)$ & $0.71(0.40-1.27)$ & $0.72(0.36-1.45)$ & $0.94(0.86-1.02)$ & 0.26 \\
\hline
\end{tabular}

HR, Hazard ratio; CI, confidence interval.

${ }^{a}$ HRs and $95 \%$ CIs per increment of 1 point at each personality subscales were calculated by treating the personality subscale (a score of $\left.0-12\right)$ as continuous variables.

${ }^{b}$ Linear trend tests were calculated by treating the personality subscales as continuous variables.

${ }^{c}$ HR1 was adjusted for age, sex, past histories of hypertension or diabetes mellitus (presence or absence), smoking status (never, former, currently smoking 1-19 cigarettes/day, or $\geqslant 20$ cigarettes/day), alcohol consumption (never, former, current ethanol intake of $<23.0$, or $\geq 23.0 \mathrm{~g} /$ day), body mass index in $\mathrm{kg} / \mathrm{m}^{2}$ ( $\leqslant 18.4,18.5-24.9$, or $\geqslant 25.0$ ), green tea consumption ( $<1 \mathrm{cup} /$ day, $1-2$ cups/day, or $\geqslant 3$ cups/day), education (in school until age $\leqslant 15$ years, age $16-18$ years, or age $\geqslant 19$ years) marital status (whether or not living with spouse), sleep duration $(\leqslant 6,7-8$, or $\geqslant 9 \mathrm{~h} /$ day), time spent walking $(<0.5,0.5-1$, or $>1 \mathrm{~h} /$ day), sense of life worth living (ikigai) (having, uncertain, or not having) and stress (high, moderate, or low).

${ }^{\mathrm{d}}$ HR2 denotes the HR1 with deaths from all-causes in the first 3 years of follow-up excluded from the analysis. 
Table 3. Hazard ratios for risk of death from suicide divided follow-up years into two separate terms (1990-1997, 1998-2008), the Miyagi Cohort, Japan, 1990-2008

\begin{tabular}{|c|c|c|c|c|c|c|c|c|c|c|c|c|c|}
\hline \multicolumn{7}{|l|}{ 1990-1997 } & \multicolumn{7}{|l|}{ 1998-2008 } \\
\hline \multirow[b]{2}{*}{ Extraversion } & \multicolumn{4}{|c|}{ Personality subscale score levels } & \multirow[t]{2}{*}{$\begin{array}{l}\text { Per } 1 \text { point } \\
\text { of score }\end{array}$} & \multirow[t]{2}{*}{$p$ trend $^{\mathrm{b}}$} & \multicolumn{5}{|c|}{ Personality subscale score levels } & \multirow[t]{2}{*}{$\begin{array}{l}\text { Per one point } \\
\text { of score }^{\mathrm{a}}\end{array}$} & \multirow[t]{2}{*}{$p$ trend $^{\mathrm{l}}$} \\
\hline & $\leqslant 3$ & $4-5$ & $6-8$ & $\geqslant 9$ & & & Extraversion & $\leqslant 3$ & $4-5$ & $6-8$ & $\geqslant 9$ & & \\
\hline No. of subjects & 8694 & 6446 & 8141 & 6151 & & & No. of subjects & 8181 & 6083 & 7627 & 5782 & & \\
\hline No. of deaths & 2 & 6 & 0 & 2 & & & No. of deaths & 21 & 19 & 22 & 18 & & \\
\hline Person-years & 64111 & 47582 & 59915 & 45372 & & & Person-years & 85095 & 63270 & 79135 & 60040 & & \\
\hline $\begin{array}{l}\text { Multivariable } \\
\text { HR1 }^{\mathrm{C}}(95 \% \mathrm{CI})\end{array}$ & 1.00 (ref.) & $\begin{array}{l}4.01 \\
\quad(0.77-20.83)\end{array}$ & N.A. & $\begin{array}{l}1.51 \\
(1.19-11.79)\end{array}$ & $\begin{array}{l}1.00 \\
(0.81-1.24)\end{array}$ & 0.63 & $\begin{array}{l}\text { Multivariable } \\
\text { HR1 }^{\mathrm{c}}(95 \% \mathrm{CI})\end{array}$ & 1.00 (ref.) & $\begin{array}{l}1.33 \\
(0.71-2.49)\end{array}$ & $\begin{array}{l}1.25 \\
(0.68-2.30)\end{array}$ & $\begin{array}{l}1.37 \\
(0.71-2.63)\end{array}$ & $\begin{array}{l}1.04 \\
(0.96-1.13)\end{array}$ & 0.29 \\
\hline Neuroticism & $\leqslant 3$ & $4-5$ & $6-7$ & $\geqslant 8$ & & & Neuroticism & $\leqslant 3$ & $4-5$ & $6-7$ & $\geqslant 8$ & & \\
\hline No. of subjects & 8495 & 6365 & 6191 & 8381 & & & No. of subjects & 8028 & 6014 & 5782 & 7849 & & \\
\hline No. of deaths & 4 & 1 & 2 & 3 & & & No. of deaths & 14 & 17 & 13 & 36 & & \\
\hline Person-years & 62794 & 46994 & 45491 & 61701 & & & Person-years & 83459 & 62530 & 60191 & 81359 & & \\
\hline $\begin{array}{l}\text { Multivariable } \\
\text { HR1 }^{\mathrm{C}}(95 \% \mathrm{CI})\end{array}$ & 1.00 (ref.) & $\begin{array}{l}0.29 \\
(0.03-2.72)\end{array}$ & $\begin{array}{l}0.50 \\
(0.08-3.02)\end{array}$ & $\begin{array}{l}0.66 \\
(0.13-3.37)\end{array}$ & $\begin{array}{l}1.01 \\
(0.81-1.26)\end{array}$ & 0.65 & $\begin{array}{l}\text { Multivariable } \\
\text { HR1 }^{\mathrm{c}}(95 \% \mathrm{CI})\end{array}$ & 1.00 (ref.) & $\begin{array}{l}1.62 \\
(0.79-3.30)\end{array}$ & $\begin{array}{l}1.23 \\
(0.57-2.65)\end{array}$ & $\begin{array}{l}2.45 \\
(1.26-4.74)\end{array}$ & $\begin{array}{l}1.13 \\
(1.04-1.23)\end{array}$ & 0.003 \\
\hline Psychoticism & $\leqslant 2$ & 3 & 4 & $\geqslant 5$ & & & Psychoticism & $\leqslant 2$ & 3 & 4 & $\geqslant 5$ & & \\
\hline No. of subjects & 9551 & 7169 & 5849 & 6863 & & & No. of subjects & 9019 & 6738 & 5510 & 6406 & & \\
\hline No. of deaths & 1 & 2 & 3 & 4 & & & No. of deaths & 16 & 12 & 15 & 37 & & \\
\hline Person-years & 70559 & 52886 & 43106 & 50428 & & & Person-years & 94187 & 70134 & 57269 & 65950 & & \\
\hline $\begin{array}{l}\text { Multivariable } \\
\text { HR1 }^{\mathrm{c}}(95 \% \mathrm{CI})\end{array}$ & 1.00 (ref.) & $\begin{array}{l}3.25 \\
(0.29-36.58)\end{array}$ & $\begin{array}{l}5.85 \\
(0.59-58.35)\end{array}$ & $\begin{array}{l}7.85 \\
(0.81-76.04)\end{array}$ & $\begin{array}{l}1.49 \\
(1.05-2.11)\end{array}$ & 0.05 & $\begin{array}{l}\text { Multivariable } \\
\text { HR1' }^{c}(95 \% \text { CI) }\end{array}$ & 1.00 (ref.) & $\begin{array}{l}0.86 \\
(0.41-1.83)\end{array}$ & $\begin{array}{l}1.17 \\
(0.57-2.38)\end{array}$ & $\begin{array}{l}2.05 \\
(1.11-3.78)\end{array}$ & $\begin{array}{l}1.13 \\
(0.99-1.30)\end{array}$ & 0.01 \\
\hline Lie & $\leqslant 5$ & $6-7$ & $8-9$ & $\geqslant 10$ & & & Lie & $\leqslant 5$ & $6-7$ & $8-9$ & $\geqslant 10$ & & \\
\hline No. of subjects & 7177 & 7378 & 8897 & 5980 & & & No. of subjects & 6700 & 6958 & 8373 & 5642 & & \\
\hline No. of deaths & 2 & 4 & 2 & 2 & & & No. of deaths & 31 & 18 & 19 & 12 & & \\
\hline Person-years & 52711 & 54480 & 65599 & 44189 & & & Person-years & 69694 & 72547 & 87188 & 58111 & & \\
\hline $\begin{array}{l}\text { Multivariable } \\
\text { HR1 }^{\mathrm{c}}(95 \% \mathrm{CI})\end{array}$ & 1.00 (ref.) & $\begin{array}{l}1.86 \\
(0.32-10.85)\end{array}$ & $\begin{array}{l}0.71 \\
(0.09-5.42)\end{array}$ & $\begin{array}{l}0.86 \\
(0.11-6.66)\end{array}$ & $\begin{array}{l}0.92 \\
(0.71-1.18)\end{array}$ & 0.58 & $\begin{array}{l}\text { Multivariable } \\
\text { HR1 }^{c}(95 \% \text { CI) }\end{array}$ & 1.00 (ref.) & $\begin{array}{l}0.71 \\
\quad(0.39-1.27)\end{array}$ & $\begin{array}{l}0.71 \\
(0.39-1.29)\end{array}$ & $\begin{array}{l}0.77 \\
(0.38-1.56)\end{array}$ & $\begin{array}{l}0.96 \\
(0.87-1.05)\end{array}$ & 0.45 \\
\hline
\end{tabular}

HR, Hazard ratio; CI, confidence interval.

${ }^{\text {a }} \mathrm{HRs}$ and $95 \%$ CIs per increment of 1 point at each personality subscales were calculated by treating the personality subscale (a score of $\left.0-12\right)$ as continuous variables.

${ }^{\mathrm{b}}$ Linear trend tests were calculated by treating the personality subscales as continuous variables.

${ }^{\mathrm{c}}$ HR1 was adjusted for age, sex, past histories of hypertension or diabetes mellitus (presence or absence), smoking status (never, former, currently smoking 1-19 cigarettes/day, or $\geqslant 20$ cigarettes/day), alcohol consumption (never, former, current ethanol intake of $<23.0$, or $\geqslant 23.0 \mathrm{~g} /$ day), body mass index in $\mathrm{kg} / \mathrm{m}^{2}$ ( $\leqslant 18.4,18.5-24.9$, or $\geqslant 25.0$ ), green tea consumption ( $<1$ cup/day, $1-2$ cups/day, or $\geqslant 3$ cups/day) education (in school until age $\leqslant 15$ years, age $16-18$ years, or age $\geqslant 19$ years) marital status (whether or not living with spouse), sleep duration $(\leqslant 6,7-8$, or $\geqslant 9 \mathrm{~h} /$ day), time spent walking $(>0.5,0.5-1$, or $>1 \mathrm{~h} /$ day), sense of life worth living (ikigai) (having, uncertain, or not having) and stress (high, moderate, or low). 


\section{Extraversion}

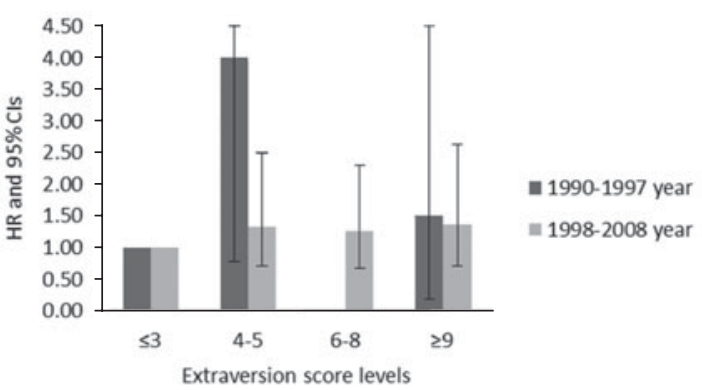

Neuroticism

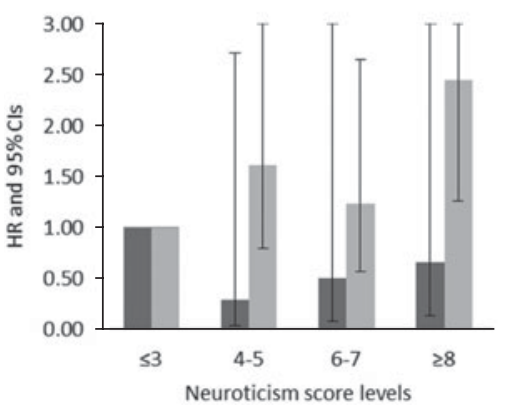

Psychoticism

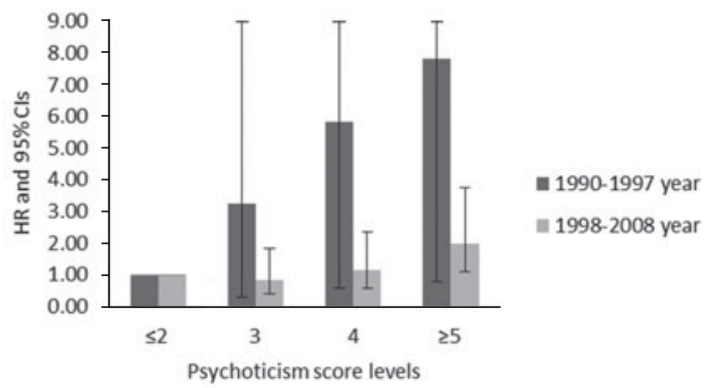

Lie

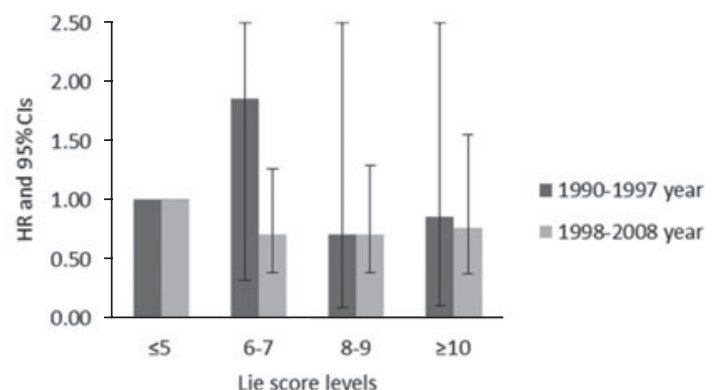

Fig. 2. Hazard ratios for risk of death from suicide divided follow-up years into two separate terms (1990-1997, 1998-2008), the Miyagi Cohort, Japan, 1990-2008.

or other), respectively, when we treated subjects who were employed by the government as a reference group.

\section{Discussion}

We investigated the association between personality and suicide risk for 19 years from 1990 to 2008 in a population-based prospective cohort study in Japan, which suffered an economic crisis in 1997-1998. We found that the suicide rate jumped in 1998, and remained high. This trend was the same as that in whole Japan (Statistics and Information Department Minister's Secretariant Ministry of Health Labour and Welfare Japan, 2011). Neuroticism and Psychoticism were significantly associated with an increased risk of suicide throughout the entire follow-up period. However, the impact of the economic crisis upon suicide risk differed between Neuroticism and Psychoticism. The suicide risk associated with Neuroticism increased after 1998, while that associated with Psychoticism decreased. The present results suggest that individuals with higher Neuroticism would be vulnerable to social stress such as economic crisis, whereas individuals with higher Psychoticism would be insensitive.

Many cross-sectional and case-control studies have reported factors associated with suicidal attempts and ideation (Park et al. 2010; Naragon-Gainey \&
Watson, 2011). The present study demonstrated for the first time the association between personality and completed suicide using a prospective approach. To our knowledge, no previous study has examined the impact of economic crisis upon the association between personality and suicide risk.

The present findings would not be explained by occupation, because occupation was not related to suicide risk in this data set. Moreover, the results cannot be explained by marital status because our data showed no association between marital status and personality.

The present finding appears to be explained by susceptibility of the subjects with a higher neuroticism score to development of depression after a major life event, which is an established risk factor for completed suicide. Many studies have shown that higher Neuroticism is associated with a higher prevalence of self-perception of mental stress. Kendler and colleagues reported that individuals with higher levels of Neuroticism were more sensitive to the depressogenic effect of adverse events than those with lower levels of Neuroticism (Kendler et al. 2004). In addition, Ormel et al. reported that stressful life events predisposed to major depression only in individuals with higher levels of Neuroticism (Ormel et al. 2001). Cox et al. suggested that Neuroticism may represent robust psychological dimensions associated with the presence 
post-traumatic stress disorder (PTSD; Cox et al. 2004). Numerous psychological autopsy studies have shown that depression is one of the strongest factors associated with completed suicides in North American and European countries, as well as in Japan (Yoshimasu et al. 2008; Hirokawa et al. 2012). Along with the above evidence, the present results can be interpreted as indicating that neuroticism was a predisposing factor for depression after the economic crisis of 1997-1998, thus causing the subsequent increase in the suicide completion.

Our study had some methodological strengths. This is the first study to investigate the interactive effects of economic crisis and personality upon suicide risk, comparing the suicide risk by personality before and after the economic crisis in 1997-1998. In addition, our sample size was sufficiently large to investigate the joint effect of economic crisis and personality upon suicide risk. This allowed us to conduct separate analyses for the periods before and after 1998 .

Our study also had several limitations. First, we had no information about potential confounders, such as the prevalence of psychiatric disorders including depression, availability of social support, and blood pressure. Second, we had no information about changes in the lifestyle habits of our study subjects during the follow-up period, some misclassification of lifestyle habits could have arisen. Third, we had no individual-level information on how the economic crisis in 1997-1998 influenced members of the cohort or how much stress each member experienced. Therefore, the possibility of ecological fallacy cannot be ruled out. Fourth, we excluded 11991 subjects out of the original cohort of 41423 due to missing in part of answers $(N=8600)$, response by other family members $(N=2493)$, and other reasons. In order to evaluate the degree of bias resulting from exclusion of the subjects for whom answers to the EPQ-R were partly missing, we conducted 'sensitivity analyses' in which we imputed the missing score for each EPQ question as either ' 0 ' or ' 1 ' to the 8600 subjects. For both Table 2 and Table 3, all three analyses (original result, missing score imputed by 0 , that imputed by 1 ) yielded similar results (data not shown). Therefore, we consider that the bias due to exclusion of subjects lacking answers was not sufficiently large to affect the present findings.

In conclusion, this study has demonstrated that, after the economic crisis in 1997-1998, the suicide mortality was increased only among those with higher Neuroticism but not other personality traits. Suicide mortality usually increases after major events such as economic crises and natural disasters. In this study, we have demonstrated that subjects with higher Neuroticism were at high risk of increased suicide mortality after the economic crisis in 1997-1998.
This finding highlights the importance of establishing a programme for identifying vulnerable subpopulations after major events (i.e. those with higher Neuroticism) and for providing them with measures for suicide prevention such as family support, peer support, psychological consultation, early detection of depressive symptoms, and proper treatment.

\section{Acknowledgements}

We thank Yoshiko Nakata, Naoko Sato, and Yumi Tamura for their technical assistance. This work was supported by a grant for Scientific Research from Ministry of Education, Culture, Sports, Science, and Technology, Japan, Grant-in-Aid for Challenging Exploratory Research (22659127) and Health Sciences Research Grant for Health Services (H23 -Junkankitou (Seisyu)-Ippan-005) from the Ministry of Health, Labour and Welfare, Japan. The founders do not bear any responsibility for the analyses or interpretations presented here.

\section{Declaration of Interest}

None.

\section{References}

Bertolote JM, Fleischmann A (2005). Suicidal behavior prevention: WHO perspectives on research. American Journal of Medical Genetics. Part C, Seminars in Medical Genetics 133C, 8-12.

Brezo J, Paris J, Turecki G (2006). Personality traits as correlates of suicidal ideation, suicide attempts, and suicide completions: a systematic review. Acta Psychiatrica Scandinavica 113, 180-206.

Chang S, Gunnell D, Sterne JAC, Lu T, Cheng ATA (2009). Was the economic crisis 1997-1998 responsible for rising suicide rates in East/Southeast Asia? A time-trend analysis for Japan, Hong Kong, South Korea, Taiwan, Singapore and Thailand. Social Science \& Medicine 68, 1322-1331.

Cox BJ, MacPherson PSR, Enns MW, McWilliams LA (2004). Neuroticism and self-criticism associated with posttraumatic stress disorder in a nationally representative sample. Behaviour Research and Therapy 42, 105-114.

Duberstein PR, Conwell Y, Caine ED (1994). Age differences in the personalitycharacteristics of suicide completers: preliminary findings from a psychological autopsy study. Psychiatry 57, 213-224.

Dupre ME (2012). The cumulative effect of unemployment on risks for acute myocardial infarction unemployment and risks for acute MI. Archives of Internal Medicine 172, 1.

Eysenck HJ, Eysenck SBG (1991). Manual of the Eysenck Personality Scales (EPS adult). Hoddder and Stoughton: London.

Fukaol A, Tsubono Y, Komatsu S, Tsuji I, Minami Y, Hisamichi S, Hosokawa T, Kawamura M, Takano A, 
Sugahara N, Ikeda T, Nishikori M (1995). A cohort study on the relation of lifestyle, personality and biologic markers to cancer in Miyagi, Japan : study design, response rate and profiles of the cohort subjects. Journal of Epidemiology 5, 153-157.

Hirokawa S, Kawakami N, Matsumoto T, Inagaki A, Eguchi N, Tsuchiya M, Katsumata Y, Akazawa M, Kameyama A, Tachimori H, Takeshima T (2012). Mental disorders and suicide in Japan: a nation-wide psychological autopsy case-control study. Journal of Affective Disorders 140, 168-175.

Hosokawa T, Ohyama M (1993). Reliability and validity of the Japanese version of the short form Eysenck personality questionnaire-revised. Psychological Reports 72, 823-832.

Inoue $K$, Tanii $H$, Kaiya $H$, Abe $S$, Nishimura $Y$, Masaki M, Okazaki Y, Nata M, Fukunaga T (2007). The correlation between unemployment and suicide rates in Japan between 1978 and 2004. Legal Medicine 9, 139-142.

Kendler KS, Kuhn J, Prescott CA (2004). The interrelationship of neuroticism, sex, and stressful life events in the prediction of episodes of major depression. American Journal of Psychiatry 161, 631-636.

Kim SY, Kim M-H, Kawachi I, Cho Y (2011). Comparative epidemiology of suicide in South Korea and Japan: effects of age, gender and suicide methods. Crisis 32, 5-14.

Lewis G, Sloggett A (1998). Suicide, deprivation, and unemployment: record linkage study. British Medical Journal $317,1283-6$.

Lolas F, Gomez A, Suarez L (1991). EPQ-R and suicide attempt: the relevance of psychoticism. Personality and Individual Differences 12, 899-902.

Nakaya N, Tsubono Y, Hosokawa T, Hozawa A, Kuriyama S, Fukudo S, Tsuji I (2005). Personality and mortality from ischemic heart disease and stroke. Clinical and Experimental Hypertension 27, 297-305.

Naragon-Gainey K, Watson D (2011). The anxiety disorders and suicidal ideation: accounting for co-morbidity via underlying personality traits. Psychological Medicine 41, 1437-47.

Ormel J, Oldehinkel AJ, Brilman EI (2001). The interplay and etiological continuity of neuroticism, difficulties, and life events in the etiology of major and subsyndromal, first and recurrent depressive episodes in later life. American Journal of Psychiatry 158, 885-891.
Park S, Cho S, Moon S (2010). Factors associated with suicidal ideation: role of emotional and instrumental support. Journal of Psychosomatic Research 69, 389-397.

Sone T, Nakaya N, Ohmori K, Shimazu T, Higashiguchi M, Kakizaki M, Kikuchi N, Kuriyama S, Tsuji I (2008). Sense of life worth living (ikigai) and mortality in Japan: Ohsaki study. Psychosomatic Medicine 70, 709-715.

Statistics and Information Department Minister's Secretariant Ministry of Health Labour and Welfare Japan (2011). Vital Statistics of Japan 2009. Health and Welfare Statistics Association: Tokyo.

Stuckler D, Basu S, Suhrcke M, Coutts A, McKee M (2009). The public health effect of economic crises and alternative policy responses in Europe: an empirical analysis. Lancet 374, 315-323.

Tsoh J, Chiu HFK, Duberstein PR, Chan SSM, Chi I, Yip PSF, Conwell Y (2005). Attempted suicide in elderly Chinese persons: a multi-group, controlled study. American Journal of Geriatric Psychiatry 13, 562-571.

Tsuji I, Nishino Y, Tsubono Y, Suzuki Y, Hozawa A, Nakaya N, Fujita K, Kuriyama S, Shibuya D, Fukao A, Hisamichi S (2004). Follow-up and mortality profiles in the Miyagi Cohort Study. Journal of Epidemiology 14 (Suppl. 1), S2-S6.

Useda JD, Duberstein PR, Conner KR, Conwell Y (2004). Personality and attempted suicide in depressed adults 50 years of age and older: a facet level analysis. Comprehensive Psychiatry 45, 353-361.

Velting DM (1999). Suicidal ideation and the five-factor model of personality. Personality and Individual Differences 27, 943-952.

Wada K, Kondo N, Gilmour S, Ichida Y, Fujino Y, Satoh T, Shibuya K (2012). Trends in cause specific mortality across occupations in Japanese men of working age during period of economic stagnation, 1980-2005: retrospective cohort study. British Medical Journal 344, e1191-e1191.

WHO (1977). Manual of the International Statistical Classification of Diseases, Injuries, and Causes of Death, 9th revision. World Health Organization: Geneva.

WHO (1992). International Statistical Classification of Diseases and Related Health Problems, 10th revision. World Health Organization: Geneva.

Yoshimasu K, Kiyohara C, Miyashita K; The Stress Research Group of the Japanese Society for Hygiene (2008). Suicidal risk factors and completed suicide: meta-analyses based on psychological autopsy studies. Environmental Health and Preventive Medicine 13, 243-256. 


\section{Appendix}

Table A1. Hazard ratios and 95\% confidence intervals for risk of death from suicide, the Miyagi Cohort, Japan, 1990-2008

\begin{tabular}{|c|c|c|c|c|c|}
\hline & \multicolumn{4}{|c|}{ Personality subscale score levels } & \multirow[b]{2}{*}{$p$ trend $^{\mathrm{a}}$} \\
\hline & $\begin{array}{l}\text { Q1 } \\
0-3\end{array}$ & $\begin{array}{l}\text { Q2 } \\
4-6\end{array}$ & $\begin{array}{l}\text { Q3 } \\
7-9\end{array}$ & $\begin{array}{l}\text { Q4 } \\
10-12\end{array}$ & \\
\hline \multicolumn{6}{|l|}{ Extraversion } \\
\hline No. of subjects & 8694 & 9461 & 7263 & 4014 & \\
\hline No. of deaths & 23 & 34 & 15 & 18 & \\
\hline Person-years & 149229 & 162174 & 124334 & 68858 & \\
\hline Age- and sex-adjusted HR (95\% CI) & 1.00 (ref.) & $1.36(0.80-2.31)$ & $0.78(0.40-1.47)$ & $1.58(0.85-2.93)$ & 0.82 \\
\hline Multivariable HR1 ${ }^{\mathrm{b}}(95 \% \mathrm{CI})$ & 1.00 (ref.) & $1.48(0.87-2.53)$ & $0.88(0.45-1.71)$ & $1.93(1.02-3.68)$ & 0.42 \\
\hline \multicolumn{6}{|l|}{ Neuroticism } \\
\hline No. of subjects & 8495 & 9579 & 7824 & 3534 & \\
\hline No. of deaths & 18 & 25 & 25 & 22 & \\
\hline Person-years & 146275 & 164128 & 134151 & 60041 & \\
\hline Age- and sex-adjusted HR (95\% CI) & 1.00 (ref.) & $1.31(0.72-2.40)$ & $1.63(0.89-2.98)$ & $3.22(1.73-6.01)$ & 0.0002 \\
\hline Multivariable HR1 $1^{\mathrm{b}}(95 \% \mathrm{CI})$ & 1.00 (ref.) & $1.22(0.66-2.26)$ & $1.45(0.77-2.71)$ & $2.77(1.40-5.49)$ & 0.003 \\
\hline \multicolumn{6}{|l|}{ Psychoticism } \\
\hline No. of subjects & 16720 & 11469 & 1214 & 29 & \\
\hline No. of deaths & 31 & 53 & 6 & 0 & \\
\hline Person-years & 287809 & 196032 & 20290 & 464 & \\
\hline Age- and sex-adjusted HR (95\% CI) & 1.00 (ref.) & $1.95(1.24-3.06)$ & $1.75(0.72-4.26)$ & N.S. & 0.02 \\
\hline Multivariable HR1 $1^{\mathrm{b}}(95 \% \mathrm{CI})$ & 1.00 (ref.) & $1.95(1.24-3.06)$ & $1.64(0.67-4.03)$ & N.S. & 0.02 \\
\hline \multicolumn{6}{|l|}{ Lie } \\
\hline No. of subjects & 2798 & 7666 & 12988 & 5980 & \\
\hline No. of deaths & 18 & 29 & 29 & 14 & \\
\hline Person-years & 47555 & 131180 & 223544 & 102315 & \\
\hline Age- and sex-adjusted HR (95\% CI) & 1.00 (ref.) & $0.68(0.38-1.23)$ & $0.46(0.25-0.84)$ & $0.50(0.24-1.04)$ & 0.07 \\
\hline Multivariable HR1 $1^{\mathrm{b}}(95 \% \mathrm{CI})$ & 1.00 (ref.) & $0.71(0.39-1.29)$ & $0.49(0.27-0.90)$ & $0.58(0.28-1.22)$ & 0.16 \\
\hline
\end{tabular}

HR, Hazard ratio; CI, confidence interval; n.s., non-significant.

${ }^{a}$ Linear trend tests were calculated by treating the personality subscales as continuous variables.

${ }^{\mathrm{b}}$ HR1 was adjusted for age, sex, past histories of hypertension or diabetes mellitus (presence or absence), smoking status (never, former, currently smoking 1-19 cigarettes/day, or $\geqslant 20$ cigarettes/day), alcohol consumption (never, former, current ethanol intake of $<23.0$, or $\geqslant 23.0 \mathrm{~g} /$ day), body mass index in $\mathrm{kg} / \mathrm{m}^{2}(\leqslant 18.4,18.5-24.9$, or $\geqslant 25.0)$, green tea consumption ( $<1$ cup/day, $1-2$ cups/day, or $\geqslant 3$ cups/day), education (in school until age $\leqslant 15$ years, age $16-18$ years, or age $\geqslant 19$ years) marital status (whether or not living with spouse), sleep duration $(\leqslant 6,7-8$, or $\geqslant 9 \mathrm{~h}$ /day), time spent walking $(<0.5,0.5-1$, or $>1 \mathrm{~h}$ /day), sense of life worth living (ikigai) (having, uncertain, or not having) and stress (high, moderate, or low). 
Table A2. Hazard ratios and 95\% confidence intervals for risk of death from suicide, where imputed missing data as ' 0 '

\begin{tabular}{|c|c|c|c|c|c|c|}
\hline \multirow[b]{2}{*}{ Extraversion } & \multicolumn{4}{|c|}{ Personality subscale score levels } & \multirow[t]{2}{*}{ Per 1 point of score ${ }^{a}$} & \multirow[t]{2}{*}{$p$ trend $^{\mathrm{b}}$} \\
\hline & $\leqslant 3$ & $4-5$ & $6-8$ & $\geqslant 9$ & & \\
\hline No. of subjects & 11183 & 8295 & 10465 & 7378 & & \\
\hline No. of deaths & 29 & 32 & 32 & 23 & & \\
\hline Person-years & 191749 & 142547 & 178793 & 126401 & & \\
\hline $\begin{array}{l}\text { Multivariable HR1 }{ }^{\mathrm{c}} \\
(95 \% \mathrm{CI})\end{array}$ & 1.00 (ref.) & $1.57(0.94-2.60)$ & $1.28(0.76-2.13)$ & $1.31(0.74-2.32)$ & $1.03(0.97-1.10)$ & 0.34 \\
\hline Neuroticism & $\leqslant 3$ & $4-5$ & $6-7$ & $\geqslant 8$ & & \\
\hline No. of subjects & 10943 & 8245 & 7896 & 10237 & & \\
\hline No. of deaths & 21 & 24 & 24 & 47 & & \\
\hline Person-years & 188347 & 141775 & 134847 & 174521 & & \\
\hline $\begin{array}{l}\text { Multivariable HR1 }{ }^{\mathrm{c}} \\
(95 \% \mathrm{CI})\end{array}$ & 1.00 (ref.) & $1.50(0.83-2.71)$ & $1.52(0.84-2.76)$ & $2.21(1.28-3.82)$ & $1.12(1.05-1.20)$ & $<0.001$ \\
\hline Psychoticism & $\leqslant 2$ & 3 & 4 & $\geqslant 5$ & & \\
\hline No. of subjects & 11782 & 9204 & 7499 & 8836 & & \\
\hline No. of deaths & 22 & 22 & 24 & 48 & & \\
\hline Person-years & 203013 & 158211 & 128267 & 149998 & & \\
\hline $\begin{array}{l}\text { Multivariable HR1 }{ }^{\mathrm{c}} \\
(95 \% \mathrm{CI})\end{array}$ & 1.00 (ref.) & $1.16(0.64-2.09)$ & $1.42(0.79-2.55)$ & $2.09(1.24-3.53)$ & $1.12(1.01-1.25)$ & 0.04 \\
\hline Lie & $\leqslant 5$ & $6-7$ & $8-9$ & $\geqslant 10$ & & \\
\hline No. of subjects & 8422 & 9247 & 11807 & 7845 & & \\
\hline No. of deaths & 41 & 27 & 28 & 20 & & \\
\hline Person-years & 143594 & 159056 & 202649 & 134190 & & \\
\hline $\begin{array}{l}\text { Multivariable HR1 }{ }^{\mathrm{c}} \\
(95 \% \mathrm{CI})\end{array}$ & 1.00 (ref.) & $0.73(0.45-1.20)$ & $0.66(0.40-1.09)$ & $0.79(0.45-1.40)$ & $0.94(0.88-1.02)$ & 0.12 \\
\hline
\end{tabular}

HR, Hazard ratio; CI, confidence interval.

${ }^{a}$ HRs and $95 \%$ CIs per increment of 1 point at each personality subscale were calculated by treating the personality subscale (a score of 0-12) as continuous variables.

${ }^{\mathrm{b}}$ Linear trend tests were calculated by treating the personality subscales as continuous variables.

${ }^{\mathrm{c}} \mathrm{HR} 1$ was adjusted for age, sex, past histories of hypertension or diabetes mellitus (presence or absence), smoking status (never, former, currently smoking 1-19 cigarettes/day, or $\geq 20$ cigarettes/day), alcohol consumption (never, former, current ethanol intake of $<23.0$, or $\geqslant 23.0 \mathrm{~g} /$ day), body mass index in $\mathrm{kg} / \mathrm{m}^{2}(\leqslant 18.4,18.5-24.9$, or $\geqslant 25.0)$, green tea consumption ( $<1$ cup/day, $1-2$ cups/day, or $\geqslant 3$ cups/day), education (in school until age $\leqslant 15$ years, age $16-18$ years, or age $\geqslant 19$ years) marital status (whether or not living with spouse), sleep duration $(\leqslant 6,7-8$, or $\geqslant 9 \mathrm{~h} /$ day), time spent walking $(<0.5,0.5-1$, or $>1 \mathrm{~h}$ /day), sense of life worth living (ikigai) (having, uncertain, or not having) and stress (high, moderate, or low). 
Table A3 Hazard ratios and 95\% confidence intervals for risk of death from suicide, where imputed missing data as ' 1 '

\begin{tabular}{|c|c|c|c|c|c|c|}
\hline \multirow[b]{2}{*}{ Extraversion } & \multicolumn{4}{|c|}{ Personality subscale score levels } & \multirow[t]{2}{*}{ Per 1 point of score ${ }^{a}$} & \multirow[t]{2}{*}{$p$ trend $^{\mathrm{b}}$} \\
\hline & $\leqslant 3$ & $4-5$ & $6-8$ & $\geqslant 9$ & & \\
\hline No. of subjects & 10645 & 8201 & 10675 & 7800 & & \\
\hline No. of deaths & 29 & 30 & 33 & 24 & & \\
\hline Person-years & 182465 & 141034 & 182536 & 133455 & & \\
\hline $\begin{array}{l}\text { Multivariable HR1 }{ }^{\mathrm{c}} \\
(95 \% \mathrm{CI})\end{array}$ & 1.00 (ref.) & $1.43(0.86-2.40)$ & $1.25(0.75-2.08)$ & $1.24(0.71-2.18)$ & $1.03(0.96-1.09)$ & 0.43 \\
\hline Neuroticism & $\leqslant 3$ & $4-5$ & $6-7$ & $\geqslant 8$ & & \\
\hline No. of subjects & 10307 & 8043 & 7943 & 11028 & & \\
\hline No. of deaths & 20 & 25 & 21 & 50 & & \\
\hline Person-years & 177583 & 138128 & 135877 & 187902 & & \\
\hline $\begin{array}{l}\text { Multivariable HR1 }{ }^{\mathrm{c}} \\
(95 \% \mathrm{CI})\end{array}$ & 1.00 (ref.) & $1.59(0.88-2.87)$ & $1.32(0.71-2.45)$ & $2.19(1.26-3.80)$ & $1.11(1.04-1.19)$ & 0.001 \\
\hline Psychoticism & $\leqslant 2$ & 3 & 4 & $\geqslant 5$ & & \\
\hline No. of subjects & 11960 & 9176 & 7426 & 8759 & & \\
\hline No. of deaths & 22 & 23 & 24 & 47 & & \\
\hline Person-years & 206121 & 157550 & 127369 & 148449 & & \\
\hline $\begin{array}{l}\text { Multivariable HR1 }{ }^{\mathrm{c}} \\
(95 \% \mathrm{CI})\end{array}$ & 1.00 (ref.) & $1.23(0.69-2.22)$ & $1.45(0.81-2.61)$ & $2.09(1.24-3.54)$ & $1.12(1.00-1.24)$ & 0.04 \\
\hline Lie & $\leqslant 5$ & $6-7$ & $8-9$ & $\geqslant 10$ & & \\
\hline No. of subjects & 8729 & 9341 & 11529 & 7722 & & \\
\hline No. of deaths & 41 & 28 & 29 & 18 & & \\
\hline Person-years & 148675 & 160531 & 198110 & 132174 & & \\
\hline $\begin{array}{l}\text { Multivariable HR1 }{ }^{\mathrm{c}} \\
(95 \% \mathrm{CI})\end{array}$ & 1.00 (ref.) & $0.78(0.48-1.27)$ & $0.72(0.44-1.19)$ & $0.74(0.41-1.33)$ & $0.95(0.88-1.02)$ & 0.13 \\
\hline
\end{tabular}

HR, Hazard ratio; CI, confidence interval.

${ }^{a}$ HRs and $95 \%$ CIs per increment of 1 point at each personality subscales were calculated by treating the personality subscale (a score of 0-12) as continuous variables.

${ }^{\mathrm{b}}$ Linear trend tests were calculated by treating the personality subscales as continuous variables.

${ }^{\mathrm{c}} \mathrm{HR} 1$ was adjusted for age, sex, past histories of hypertension or diabetes mellitus (presence or absence), smoking status (never, former, currently smoking 1-19 cigarettes/day, or $\geqslant 20$ cigarettes/day), alcohol consumption (never, former, current ethanol intake of $<23.0$, or $\geqslant 23.0 \mathrm{~g} /$ day), body mass index in $\mathrm{kg} / \mathrm{m}^{2}(\leqslant 18.4,18.5-24.9$, or $\geqslant 25.0)$, green tea consumption ( $<1$ cup/day, $1-2$ cups/day, or $\geqslant 3$ cups/day), education (in school until age $\leqslant 15$ years, age $16-18$ years, or age $\geqslant 19$ years) marital status (whether or not living with spouse), sleep duration $(\leqslant 6,7-8$, or $\geqslant 9 \mathrm{~h}$ /day), time spent walking $(<0.5,0.5-1$, or $>1 \mathrm{~h}$ /day), sense of life worth living (ikigai) (having, uncertain, or not having) and stress (high, moderate, or low). 


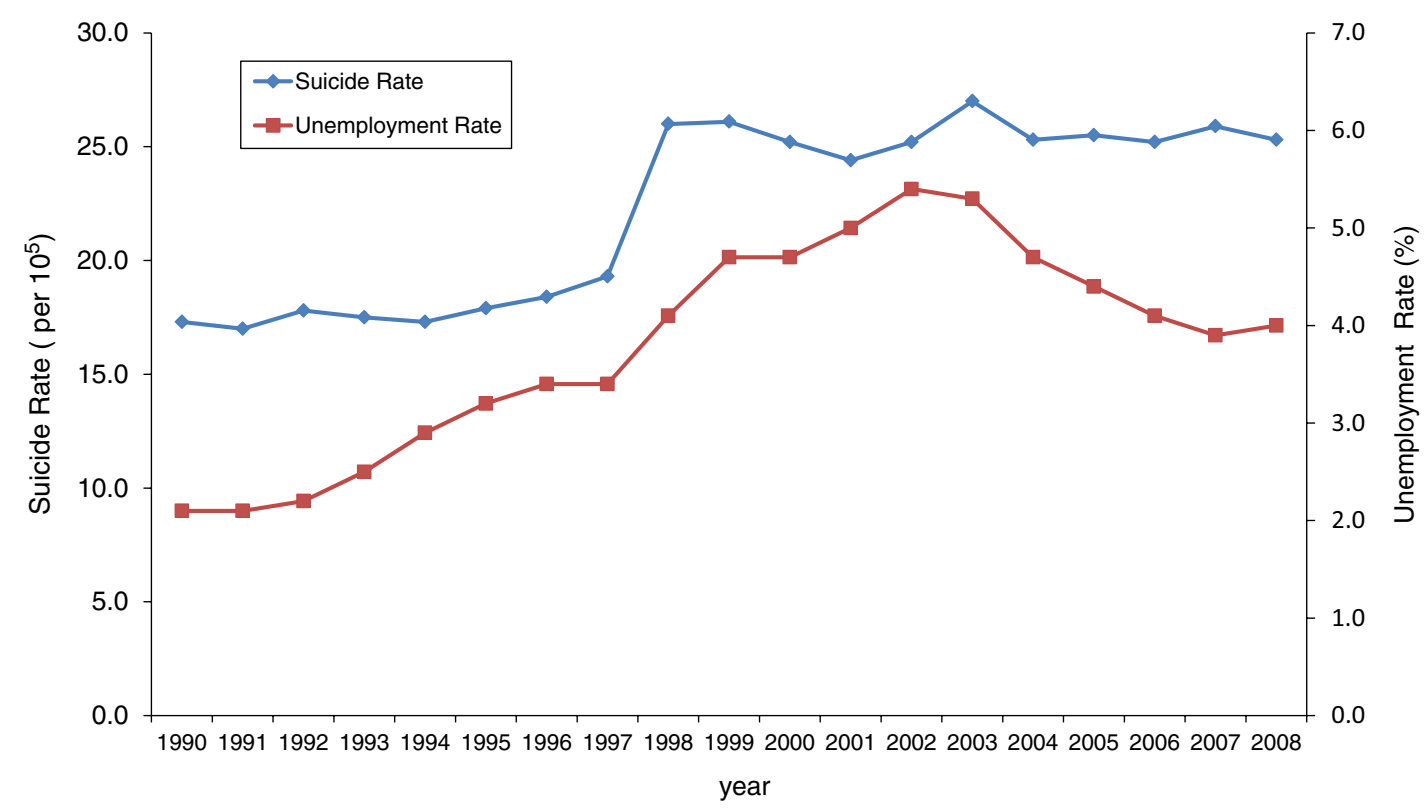

Fig. A1. Suicide rate and unemployment rate in Japan, 1990-2008.

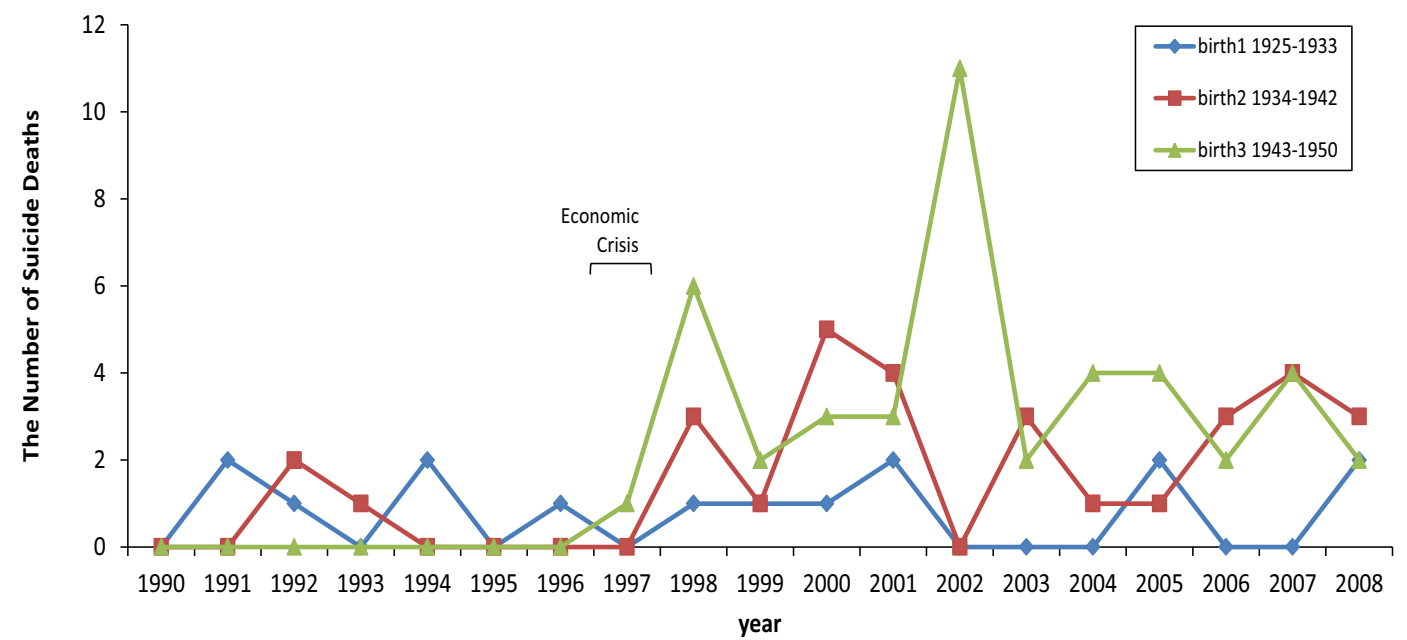

Fig. A2. Number of suicide deaths in the subjects of Miyagi Cohort Study 1990-2008. 